\title{
Acoustical Behaviour of Sodium Nitroprusside in Aquo-Organic Solvent Media at 308.15 K
}

\author{
Monalisa Das, Smrutiprava Das, and A. K. Pattanaik \\ Department of Chemistry, Ravenshaw University, College Square, Odisha, Cuttack 753003, India \\ Correspondence should be addressed to Smrutiprava Das; dassmrutiprava@yahoo.in
}

Received 17 June 2012; Revised 5 September 2012; Accepted 19 September 2012

Academic Editor: Yenamandra S. Prabhakar

Copyright (C) 2013 Monalisa Das et al. This is an open access article distributed under the Creative Commons Attribution License, which permits unrestricted use, distribution, and reproduction in any medium, provided the original work is properly cited.

Density and ultrasonic velocity have been measured for sodium nitroprusside in aqueous solutions of $\mathrm{CH}_{3} \mathrm{OH}$, ethylene glycol, DMSO, and n-propanol solvents at $308.15 \mathrm{~K}$. A quantitative relationship has been established among the acoustical properties like ultrasonic velocity $(U)$, adiabatic compressibility $(\beta)$, intermolecular free length $\left(L_{f}\right)$, acoustic impedance $(Z)$, apparent molar compressibility $\left(K_{\phi}\right)$, apparent molar volume $\left(V_{\phi}\right)$, limiting apparent molar compressibility $\left(K_{\phi}^{0}\right)$ limiting apparent molar volume $\left(V_{\phi}^{0}\right)$, and their constants $\left(S_{K}, S_{v}\right)$. From the obtained values, molecular interaction study has been made successfully in the light of these acoustical properties through hydrogen bonding in solute and solvent mixture.

\section{Introduction}

In the recent years, ultrasonic velocity measurements are helpful to interpret solute-solvent, ion-solvent, and solventsolvent interactions in aqueous and nonaqueous medium [14]. Recently, acoustic parameter studies of metal complexes have been carried out in our laboratory [5], and A. P. Mishra and D. K. Mishra have also reported a similar work [6]. The interaction helps in better understanding the types of solute and solvent, that is, whether the added solute modifies or distorts the structure of the solvent. Apparent molar volume gives valuable information about ion-ion and ion-solvent interactions in solution [7-11]. The addition of organic solvent to an aqueous solvent of electrolyte brings about a change in ion solvation that results in a large change in the reactivity of dissolved electrolyte $[12,13]$. The transition metal plays a vital role in life system because of the natural presence in vitamins, proteins, and enzymes. Sodium nitroprusside (SNP), an Fe (II) ion complex, is an effective drug which rapidly lowers blood pressure and causes vascular smooth muscle relaxation. It is also used in a urine analysis test. In the presence of buffers, it is used as a reagent for ketone strips which test the ketone level in the urine of a diabetic. It is used by forensic chemists in Simons being fast for the identification of illicit substances [14]. Thus, an attempt has been made to elucidate the ion-ion interaction between nitroprusside ions and ion-solvent interaction of SNP in aqueous solutions of methanol, ethylene glycol, npropanol, and DMSO at $308.15 \mathrm{~K}$ through ultrasonic velocity and density measurement.

\section{Experimental}

The entire chemicals used in this present research work are spectroscopic reagents (SRs), analytical reagent (AR) grades of minimum assay of $99.9 \%$ obtained from E-Merck, Germany, and SD fine chemicals, India, which are used without further purification. Water used in these experiments was deionized and distilled. A synthetic method of preparation of SNP has been reported [15]. The required quantity of SNP was dissolved in binary mixture of aqueous methanol, ethylene glycol, DMSO, and n-propanol, and a similar procedure has been adopted for different molalities of SNP. The density was determined using a specific gravity bottle by a relative measurement method. The ultrasonic velocity was measured by ultrasonic interferometer having frequency $2 \mathrm{MHz}$ (Mittal Enterprises, model no. F-81). The constant temperature is mentioned by circulatory water through the double wall measuring cell made up of steel. 


\section{Theory}

Using the measured data, various acoustic parameters such as adiabatic compressibility, intermolecular free length, acoustic impedance, apparent molar compressibility, apparent molar volume, limiting apparent molar compressibility, limiting apparent molar volume, and the associated constants $S_{K}$ and $S_{V}$ are calculated.

Adiabatic compressibility $(\beta)=1 /\left(U^{2} d\right)$.

Relative association can be fitted to an equation of the form

$$
R_{A}=\left(\frac{d}{d_{0}}\right) \times\left(\frac{U_{0}}{U}\right)^{1 / 3} .
$$

Acoustic impedance, $Z=U \times d$.

Intermolecular free length can be determined by

$$
L_{f}=K_{j} \beta^{1 / 2}
$$

where $K_{j}$ is Jacobson constant $=2.0965 \times 10^{-6}$.

The apparent molar compressibility, $K_{\phi}$, can be computed from

$$
K_{\phi}=100 \beta m^{-1}-\beta^{0} d^{-1}\left(1000 m^{-1}-M_{2}\right) .
$$

From the density data, the apparent molar volume $V_{\phi}$ was calculated from the relation

$$
V_{\phi}=1000\left(m d_{0}\right)^{-1}\left(d_{0}-d\right)+M_{2} d_{0}^{-1} .
$$

The apparent molar compressibility $\left(K_{\phi}\right)$ and the apparent molar volume $\left(V_{\phi}\right)$ thus obtained are found to vary linearly with $m^{1 / 2}$. The $K_{\phi}$ and $V_{\phi}$ data were fitted by least squares method

$$
\begin{aligned}
& K_{\phi}=K_{\phi}^{0}+S_{K} m^{1 / 2}, \\
& V_{\phi}=V_{\phi}^{0}+S_{V} m^{1 / 2},
\end{aligned}
$$

where $K_{\phi}^{0}$ and $V_{\phi}^{0}$ are limiting apparent molar compressibility and limiting apparent molar volume, respectively. $S_{K}$ and $S_{V}$ are the slopes.

\section{Result and Discussion}

The desired parameters such as adiabatic compressibility $(\beta)$, intermolecular free length $\left(L_{f}\right)$, acoustic impedance $(Z)$, relative association $\left(R_{A}\right)$, apparent molar comparability $\left(K_{\phi}\right)$, apparent molar volume $\left(V_{\phi}\right)$, limiting apparent molar compressibility $\left(K_{\phi}^{0}\right)$, limiting apparent molar volume $\left(V_{\phi}^{0}\right)$ and their constants $\left(S_{K}, S_{V}\right)$ have been studied at $308.15 \mathrm{~K}$. The density increases with the increase in the concentration of sodium nitroprusside due to the increased electrostriction in the system. The density increases in case of $\mathrm{CH}_{3} \mathrm{OH}+$ water, ethylene glycol + water, DMSO + water, and npropanol + water with the increase in the concentration of sodium nitroprusside, but it decreases with mole fraction of $\mathrm{CH}_{3} \mathrm{OH}$ and n-propanol and increases with the mole fraction of ethylene glycol and DMSO for a particular concentration of sodium nitroprusside. The decrease in density in case of $\mathrm{CH}_{3} \mathrm{OH}+$ water and n-propanol + water indicates the decrease in ion-solvent interaction or structure-breaking properties of sodium introprusside. But addition of ethylene glycol and DMSO to water has rigidified the threedimensional structure of water forming a strong hydrogen bond between solvent moleuculs [16, 17]. It also indicates the structure-forming properties of sodium nitroprusside in ethylene glycol + water and DMSO + water.

Table 1 shows that ultrasonic velocity increases with the increase in the concentration of sodium nitroprusside in aqueous $\mathrm{CH}_{3} \mathrm{OH}$ and DMSO, but it is found to be decreased in aqueous ethylene glycol and n-propanol. Molecular association is responsible for the observed increase in ultrasonic velocity in these mixtures. The increase in ultrasonic velocity in these solutions may be attributed to the cohesion brought about by the hydration [18].

The adiabatic compressibility (Table 1) decreased with increasing the concentration of solute in all aqueous solvent solutions except n-propanol + water. The decreasing adiabatic compressibility observed for sodium introprusside in $\mathrm{CH}_{3} \mathrm{OH}+$ water, ethylene glycol + water, and DMSO + water confirms the calculation drawn from the velocity data. The increasing electrostrictive compression of water around the solute molecules results in a large decrease in the compressibility of solutions. The decrease in the compressibility implies that the molecular association is enhanced in these systems with the increase in the solute content as the new entities formed due to molecular association become compact and less compressable [19]. The compressibility appeared to decrease with increasing hydrogen bond strength between solute and solvent molecules. The larger decrease in compressibility of nitroprusside in DMSO + water indicates larger molecular association capacity than other solvents. But the increase of adiabatic compressibility with the increase in concentration of solution may be due to collection of solvent molecules around ion [20] supporting weak ionsolvent interaction. This indicates that there is significant ionsolvent interaction.

The intermolecular free length $\left(L_{f}\right)$ is a predominant factor in solvation chemistry. Intermolecular free length depends on the intermolecular attractive and repulsive forces. In the present paper, the intermolecular free length is found to decrease with the increase in the concentration of nitroprusside as shown in Table 1, indicating a significant molecular association between solute and solvent molecules, suggesting a structure-promoting behaviors on addition of solute.

The character that determines the restriction/backward movement of sound wave is known as acoustic impendence $(Z)$. It has been estimated for nitroprusside solution of $\mathrm{CH}_{3} \mathrm{OH}+$ water, ethylene glycol + water, and DMSO + water which is found to increase with the increase in the concentration of sodium nitroprusside. But a reverse trend in the value of $Z$ is observed for sodium nitroprusside solution of n-propanol + water. The higher impedance in DMSO + water indicates the presence of bulkier-solvated ion due to ion-solvent/solvent-solvent interaction which restrict the free flow of ultrasonic velocity. 
TABLE 1: The values of density $(d)$, ultrasonic velocity $(U)$, adiabatic compressibility $(\beta)$, intermolecular free length $\left(L_{f}\right)$, acoustic impedance $(Z)$, and relative association $\left(R_{A}\right)$ of sodium nitroprusside in $\mathrm{CH}_{3} \mathrm{OH}+$ water, ethylene glycol + water, DMSO + water, and n-propanol + water at $308.15 \mathrm{~K}$.

\begin{tabular}{|c|c|c|c|c|c|c|c|}
\hline $\begin{array}{l}\text { Mole fraction } \\
\text { (Xorg) } \\
\end{array}$ & $\begin{array}{c}\text { Concentration } \\
(\mathrm{m}) \\
\mathrm{mol} \mathrm{dm}^{-3} \\
\end{array}$ & $\begin{array}{c}\text { Density } \\
(d) \\
\left(10^{3} \mathrm{~kg} / \mathrm{m}^{3}\right) \\
\end{array}$ & $\begin{array}{c}\text { Ultrasonic } \\
\text { velocity } \\
(U)(\mathrm{m} / \mathrm{sec})\end{array}$ & $\begin{array}{c}\text { Adiabatic } \\
\text { compressibility } \\
(\beta) \times 10^{-7} \mathrm{~N}^{-1} \mathrm{~m}^{2} \\
\end{array}$ & $\begin{array}{l}\text { Intermolecular } \\
\text { free length } \\
\left(L_{f}\right) \times 10^{-13} \mathrm{~m} \\
\end{array}$ & $\begin{array}{c}\text { Acoustic } \\
\text { impedance } \\
(Z) \times 10^{-3} \mathrm{~kg} \mathrm{~m}^{-2} \mathrm{~s}^{-1}\end{array}$ & $\begin{array}{c}\text { Relative } \\
\text { association } \\
\left(R_{A}\right) \\
\end{array}$ \\
\hline \multicolumn{8}{|c|}{ Sodium nitroprusside $+\mathrm{CH}_{3} \mathrm{OH}+$ water } \\
\hline \multirow{5}{*}{0.047} & 0.01 & 0.9818 & 1532.4 & 4.3374 & 1.3807 & 1.5045 & 0.9824 \\
\hline & 0.02 & 0.9827 & 1534.4 & 4.3221 & 1.3783 & 1.5078 & 0.9829 \\
\hline & 0.03 & 0.9837 & 1536.6 & 4.3065 & 1.3758 & 1.5115 & 0.9834 \\
\hline & 0.04 & 0.9846 & 1538.7 & 4.2897 & 1.3731 & 1.5147 & 0.9838 \\
\hline & 0.05 & 0.9855 & 1540.4 & 4.2763 & 1.3710 & 1.5180 & 0.9844 \\
\hline \multirow{5}{*}{0.099} & 0.01 & 0.9696 & 1552.3 & 4.2801 & 1.3716 & 1.5051 & 0.9660 \\
\hline & 0.02 & 0.9705 & 1553.6 & 4.2690 & 1.3698 & 1.5077 & 0.9666 \\
\hline & 0.03 & 0.9715 & 1554.8 & 4.2580 & 1.3680 & 1.5104 & 0.9674 \\
\hline & 0.04 & 0.9724 & 1538.7 & 4.2470 & 1.3663 & 1.5131 & 0.9680 \\
\hline & 0.05 & 0.9733 & 1540.4 & 4.2360 & 1.3645 & 1.5158 & 0.9686 \\
\hline \multirow{5}{*}{0.160} & 0.01 & 0.9574 & 1573.3 & 4.2203 & 1.3620 & 1.5061 & 0.9496 \\
\hline & 0.02 & 0.9583 & 1573.7 & 4.2136 & 1.3609 & 1.5080 & 0.9495 \\
\hline & 0.03 & 0.9588 & 1574.3 & 4.2082 & 1.3600 & 1.5094 & 0.9508 \\
\hline & 0.04 & 0.9597 & 1574.8 & 4.2016 & 1.3589 & 1.5113 & 0.9516 \\
\hline & 0.05 & 0.9602 & 1575.5 & 4.1957 & 1.3580 & 1.5127 & 0.9519 \\
\hline \multirow{5}{*}{0.228} & 0.01 & 0.9424 & 1556.3 & 4.3810 & 1.3800 & 1.4666 & 0.9381 \\
\hline & 0.02 & 0.9433 & 1555.9 & 4.3791 & 1.3875 & 1.4676 & 0.9391 \\
\hline & 0.03 & 0.9436 & 1555.6 & 4.3794 & 1.3874 & 1.4678 & 0.9394 \\
\hline & 0.04 & 0.9442 & 1555.2 & 4.3789 & 1.3873 & 1.4684 & 0.9401 \\
\hline & 0.05 & 0.9452 & 1554.9 & 4.3759 & 1.3868 & 1.4696 & 0.9412 \\
\hline \multirow{5}{*}{0.307} & 0.01 & 0.9273 & 1537.7 & 4.5608 & 1.4158 & 1.4259 & 0.9268 \\
\hline & 0.02 & 0.9276 & 1536.7 & 4.5652 & 1.4165 & 1.4257 & 0.9273 \\
\hline & 0.03 & 0.9283 & 1535.6 & 4.5683 & 1.4170 & 1.4254 & 0.9282 \\
\hline & 0.04 & 0.9286 & 1534.6 & 4.5728 & 1.4177 & 1.4250 & 0.9287 \\
\hline & 0.05 & 0.9292 & 1533.5 & 4.5764 & 1.4183 & 1.4249 & 0.9295 \\
\hline \multicolumn{8}{|c|}{ Sodium nitroprusside + ethylene glycol + water } \\
\hline \multirow{5}{*}{0.035} & 0.01 & 1.0071 & 1542.0 & 4.1760 & 1.3548 & 1.5529 & 1.0056 \\
\hline & 0.02 & 1.0081 & 1541.4 & 4.1751 & 1.3547 & 1.5538 & 1.0067 \\
\hline & 0.03 & 1.0086 & 1540.8 & 4.1763 & 1.3548 & 1.5540 & 1.0074 \\
\hline & 0.04 & 1.0093 & 1540.2 & 4.1766 & 1.3549 & 1.5544 & 1.0082 \\
\hline & 0.05 & 1.0099 & 1539.6 & 4.1774 & 1.3550 & 1.5548 & 1.0089 \\
\hline \multirow{5}{*}{0.075} & 0.01 & 1.0165 & 1572.3 & 3.9794 & 1.3225 & 1.5982 & 1.0184 \\
\hline & 0.02 & 1.0175 & 1571.7 & 3.9786 & 1.3224 & 1.5992 & 1.0095 \\
\hline & 0.03 & 1.0185 & 1571.0 & 3.9782 & 1.3223 & 1.6000 & 1.0107 \\
\hline & 0.04 & 1.0193 & 1570.3 & 3.9786 & 1.3220 & 1.6006 & 1.0116 \\
\hline & 0.05 & 1.0203 & 1569.6 & 3.9783 & 1.3198 & 1.6014 & 1.0128 \\
\hline \multirow{5}{*}{0.121} & 0.01 & 1.0259 & 1610.8 & 3.7567 & 1.2850 & 1.6525 & 1.0096 \\
\hline & 0.02 & 1.0274 & 1612.3 & 3.7443 & 1.2829 & 1.6564 & 1.0107 \\
\hline & 0.03 & 1.0287 & 1613.7 & 3.7331 & 1.2809 & 1.6600 & 1.0117 \\
\hline & 0.04 & 1.0301 & 1615.2 & 3.7211 & 1.2789 & 1.6638 & 1.0182 \\
\hline & 0.05 & 1.0315 & 1617.5 & 3.7055 & 1.2762 & 1.6684 & 1.0137 \\
\hline \multirow{5}{*}{0.177} & 0.01 & 1.0362 & 1636.5 & 3.6035 & 1.2585 & 1.6957 & 1.0143 \\
\hline & 0.02 & 1.0381 & 1637.7 & 3.5916 & 1.2564 & 1.7000 & 1.0160 \\
\hline & 0.03 & 1.0390 & 1638.8 & 3.5837 & 1.2550 & 1.7027 & 1.0166 \\
\hline & 0.04 & 1.0409 & 1639.9 & 3.5724 & 1.2531 & 1.7069 & 1.0192 \\
\hline & 0.05 & 1.0419 & 1641.4 & 3.5637 & 1.2515 & 1.7098 & 1.0190 \\
\hline
\end{tabular}


TABle 1: Continued.

\begin{tabular}{|c|c|c|c|c|c|c|c|}
\hline $\begin{array}{l}\text { Mole fraction } \\
\text { (Xorg) }\end{array}$ & $\begin{array}{l}\text { Concentration } \\
\qquad(\mathrm{m}) \\
\mathrm{mol} \mathrm{dm}^{-3}\end{array}$ & $\begin{array}{c}\text { Density } \\
(d) \\
\left(10^{3} \mathrm{~kg} / \mathrm{m}^{3}\right)\end{array}$ & $\begin{array}{c}\text { Ultrasonic } \\
\text { velocity } \\
(U)(\mathrm{m} / \mathrm{sec})\end{array}$ & $\begin{array}{c}\text { Adiabatic } \\
\text { compressibility } \\
(\beta) \times 10^{-7} \mathrm{~N}^{-1} \mathrm{~m}^{2}\end{array}$ & $\begin{array}{l}\text { Intermolecular } \\
\text { free length } \\
\left(L_{f}\right) \times 10^{-13} \mathrm{~m}\end{array}$ & $\begin{array}{c}\text { Acoustic } \\
\text { impedance } \\
(Z) \times 10^{-3} \mathrm{~kg} \mathrm{~m}^{-2} \mathrm{~s}^{-1}\end{array}$ & $\begin{array}{c}\text { Relative } \\
\text { association } \\
\left(R_{A}\right)\end{array}$ \\
\hline \multirow{5}{*}{0.243} & 0.01 & 1.0475 & 1663.6 & 3.4494 & 1.2313 & 1.7457 & 1.0198 \\
\hline & 0.02 & 1.0494 & 1664.0 & 3.4415 & 1.2299 & 1.7462 & 1.0216 \\
\hline & 0.03 & 1.0503 & 1664.4 & 3.4369 & 1.2291 & 1.7481 & 1.0224 \\
\hline & 0.04 & 1.0522 & 1664.8 & 3.4291 & 1.2277 & 1.7517 & 1.0241 \\
\hline & 0.05 & 1.0541 & 1665.2 & 3.4213 & 1.2263 & 1.7552 & 1.0259 \\
\hline \multicolumn{8}{|c|}{ Sodium nitroprusside + DMSO + water } \\
\hline \multirow{5}{*}{0.027} & 0.01 & 1.0071 & 1553.5 & 4.1144 & 1.3448 & 1.5645 & 1.0031 \\
\hline & 0.02 & 1.0087 & 1554.0 & 4.1052 & 1.3433 & 1.5675 & 1.0046 \\
\hline & 0.03 & 1.0099 & 1554.4 & 4.0982 & 1.3421 & 1.5697 & 1.0057 \\
\hline & 0.04 & 1.0109 & 1554.9 & 4.0915 & 1.3410 & 1.5718 & 1.0065 \\
\hline & 0.05 & 1.0137 & 1555.4 & 4.0776 & 1.3387 & 1.5767 & 1.0093 \\
\hline \multirow{5}{*}{0.059} & 0.01 & 1.0203 & 1590.2 & 3.8759 & 1.0352 & 1.6224 & 1.0084 \\
\hline & 0.02 & 1.0212 & 1591.1 & 3.8676 & 1.3038 & 1.6248 & 1.0091 \\
\hline & 0.03 & 1.0230 & 1592.0 & 3.8569 & 1.3020 & 1.6286 & 1.0107 \\
\hline & 0.04 & 1.0240 & 1592.9 & 3.8488 & 1.3006 & 1.6311 & 1.0115 \\
\hline & 0.05 & 1.0259 & 1593.8 & 3.8373 & 1.2987 & 1.6350 & 1.0132 \\
\hline \multirow{5}{*}{0.097} & 0.01 & 1.0334 & 1628.1 & 3.6506 & 1.2667 & 1.6824 & 1.0133 \\
\hline & 0.02 & 1.0344 & 1628.8 & 3.6440 & 1.2656 & 1.6848 & 1.0142 \\
\hline & 0.03 & 1.0362 & 1629.6 & 3.6341 & 1.2638 & 1.6886 & 1.0158 \\
\hline & 0.04 & 1.0372 & 1630.4 & 3.6270 & 1.2626 & 1.6910 & 1.0166 \\
\hline & 0.05 & 1.0381 & 1631.1 & 3.6208 & 1.2615 & 1.6932 & 1.0173 \\
\hline \multirow{5}{*}{0.144} & 0.01 & 1.0466 & 1656.1 & 3.4837 & 1.2374 & 1.7332 & 1.0205 \\
\hline & 0.02 & 1.0475 & 1657.0 & 3.4379 & 1.2293 & 1.7351 & 1.0212 \\
\hline & 0.03 & 1.0484 & 1658.0 & 3.4245 & 1.2269 & 1.7382 & 1.0218 \\
\hline & 0.04 & 1.0503 & 1658.9 & 3.4179 & 1.2257 & 1.7423 & 1.0235 \\
\hline & 0.05 & 1.0512 & 1659.8 & 3.4531 & 1.2320 & 1.7447 & 1.0242 \\
\hline \multirow{5}{*}{0.307} & 0.01 & 1.0606 & 1683.3 & 3.3276 & 1.2094 & 1.7853 & 1.0285 \\
\hline & 0.02 & 1.0613 & 1684.2 & 3.3218 & 1.2083 & 1.7874 & 1.0290 \\
\hline & 0.03 & 1.0619 & 1685.0 & 3.3168 & 1.2074 & 1.7893 & 1.0294 \\
\hline & 0.04 & 1.0625 & 1685.9 & 3.3114 & 1.2064 & 1.7912 & 1.0298 \\
\hline & 0.05 & 1.0634 & 1686.8 & 3.3050 & 1.2053 & 1.7937 & 1.0305 \\
\hline \multicolumn{8}{|c|}{ Sodium nitroprusside + n-propanol + water } \\
\hline \multirow{5}{*}{0.206} & 0.01 & 0.9893 & 1681.3 & 3.5759 & 1.2537 & 1.6633 & 0.9598 \\
\hline & 0.02 & 0.9902 & 1678.8 & 3.5833 & 1.2550 & 1.6623 & 0.9611 \\
\hline & 0.03 & 0.9912 & 1676.1 & 3.5912 & 1.2564 & 1.6563 & 0.9626 \\
\hline & 0.04 & 0.9921 & 1661.3 & 3.6521 & 1.2670 & 1.6482 & 0.9663 \\
\hline & 0.05 & 0.9931 & 1658.1 & 3.6626 & 1.2688 & 1.6467 & 0.9679 \\
\hline \multirow{5}{*}{0.057} & 0.01 & 0.9715 & 1627.2 & 3.8875 & 1.3071 & 1.5808 & 0.9528 \\
\hline & 0.02 & 0.9724 & 1621.4 & 3.9118 & 1.3072 & 1.5766 & 0.9548 \\
\hline & 0.03 & 0.9733 & 1625.8 & 3.8870 & 1.3112 & 1.5724 & 0.9549 \\
\hline & 0.04 & 0.9743 & 1609.4 & 3.9626 & 1.3197 & 1.5680 & 0.9591 \\
\hline & 0.05 & 0.9451 & 1605.3 & 3.9796 & 1.3226 & 1.5653 & 0.9607 \\
\hline \multirow{5}{*}{0.094} & 0.01 & 0.9546 & 1573.5 & 4.2310 & 1.3637 & 1.5021 & 0.9468 \\
\hline & 0.02 & 0.9555 & 1568.5 & 4.2540 & 1.3674 & 1.4987 & 0.9486 \\
\hline & 0.03 & 0.9564 & 1563.4 & 4.2778 & 1.3712 & 1.4952 & 0.9506 \\
\hline & 0.04 & 0.9578 & 1558.5 & 4.2984 & 1.3745 & 1.4927 & 0.9530 \\
\hline & 0.05 & 0.9583 & 1553.0 & 4.3267 & 1.3790 & 1.4882 & 0.9546 \\
\hline
\end{tabular}


TABLE 1: Continued.

\begin{tabular}{|c|c|c|c|c|c|c|c|}
\hline Mole fraction & $\begin{array}{l}\text { Concentration } \\
\qquad(\mathrm{m}) \\
\text { mol dm }\end{array}$ & $\begin{array}{c}\text { Density } \\
(d) \\
\left(10^{3} \mathrm{~kg} / \mathrm{m}^{3}\right)\end{array}$ & $\begin{array}{l}\text { Ultrasonic } \\
\text { velocity } \\
(U)(\mathrm{m} / \mathrm{sec})\end{array}$ & $\begin{array}{c}\text { Adiabatic } \\
\text { compressibility } \\
(\beta) \times 10^{-7} \mathrm{~N}^{-1} \mathrm{~m}^{2}\end{array}$ & $\begin{array}{l}\text { Intermolecular } \\
\text { free length } \\
\left(L_{f}\right) \times 10^{-13} \mathrm{~m}\end{array}$ & $\begin{array}{c}\text { Acoustic } \\
\text { impedance } \\
(Z) \times 10^{-3} \mathrm{~kg} \mathrm{~m}^{-2} \mathrm{~s}^{-1}\end{array}$ & $\begin{array}{c}\text { Relative } \\
\text { association } \\
\left(R_{A}\right)\end{array}$ \\
\hline \multirow{5}{*}{0.138} & 0.01 & 0.9367 & 1517.7 & 4.6368 & 1.4246 & 1.4216 & 0.9403 \\
\hline & 0.02 & 0.9386 & 1515.3 & 4.6410 & 1.4281 & 1.4223 & 0.9427 \\
\hline & 0.03 & 0.9396 & 1509.8 & 4.6689 & 1.4325 & 1.4186 & 0.9448 \\
\hline & 0.04 & 0.9405 & 1504.3 & 4.6986 & 1.4371 & 1.4145 & 0.9469 \\
\hline & 0.05 & 0.9424 & 1499.6 & 4.7186 & 1.4401 & 1.4132 & 0.9498 \\
\hline \multirow{5}{*}{0.194} & 0.01 & 0.9198 & 1464.4 & 5.0698 & 1.4928 & 1.3469 & 0.9344 \\
\hline & 0.02 & 0.9208 & 1461.1 & 5.0872 & 1.4953 & 1.3454 & 0.9361 \\
\hline & 0.03 & 0.9227 & 1457.8 & 5.0997 & 1.4972 & 1.3451 & 0.9381 \\
\hline & 0.04 & 0.9245 & 1457.8 & 5.1143 & 1.4993 & 1.3445 & 0.9413 \\
\hline & 0.05 & 0.9264 & 1451.0 & 5.1270 & 1.5012 & 1.3442 & 0.9440 \\
\hline
\end{tabular}

Xorg: mole fractions of the organic cosolvent, corresponding to Xorg, are 10, 20, 30, 40, and $50 \mathrm{v} / \mathrm{V} \%$, respectively.

TABLE 2: The data of concentration, apparent molar compressibility $\left(K_{\phi}\right)$, and apparent molar volume $\left(V_{\phi}\right)$ of sodium nitroprusside in $\mathrm{CH}_{3} \mathrm{OH}+$ water, ethylene glycol + water, DMSO + water, and n-propanol + water at $308.15 \mathrm{~K}$.

\begin{tabular}{|c|c|c|c|c|c|c|c|c|c|c|}
\hline $\begin{array}{l}\text { Concentration } \\
(\mathrm{m}) \mathrm{mol} \mathrm{dm}^{-3}\end{array}$ & \multicolumn{5}{|c|}{$\begin{array}{l}\text { Apparent molar compressibility } \\
\qquad-\left(K_{\phi}\right) \times 10^{-3} \mathrm{~m}^{2} \mathrm{~N}^{-1}\end{array}$} & \multicolumn{5}{|c|}{$\begin{array}{l}\text { Apparent molar volume } \\
\left(V_{\phi}\right) \times 10^{-3} \mathrm{~m}^{3} \mathrm{~mol}^{-1}\end{array}$} \\
\hline \multicolumn{11}{|c|}{ Sodium nitroprusside $+\mathrm{CH}_{3} \mathrm{OH}+$ water } \\
\hline & & & & & Xorg & & & & & \\
\hline & 0.047 & 0.099 & 0.160 & 0.228 & 0.307 & 0.047 & 0.099 & 0.160 & 0.228 & 0.307 \\
\hline 0.01 & 0.8895 & 1.7665 & 1.8060 & 2.2354 & 1.5545 & 1.2153 & 1.8549 & 2.7133 & 3.4489 & 4.5363 \\
\hline 0.02 & 0.4551 & 1.4259 & 1.3810 & 1.7833 & 1.0767 & 0.8676 & 1.6813 & 2.0950 & 2.9807 & 3.9907 \\
\hline 0.03 & 0.3113 & 1.1368 & 0.9367 & 1.3445 & 0.7567 & 0.6448 & 1.3115 & 1.6218 & 1.9895 & 2.6502 \\
\hline 0.04 & 0.2424 & 0.7206 & 0.6381 & 0.9238 & 0.4653 & 0.5359 & 1.1053 & 1.3798 & 1.5520 & 2.2165 \\
\hline 0.05 & 0.1329 & 0.4721 & 0.3549 & 0.8459 & 0.3059 & 0.4705 & 0.8742 & 1.2162 & 1.2814 & 1.6033 \\
\hline
\end{tabular}

Sodium nitroprusside + ethylene glycol + water

\begin{tabular}{ccccccccccc}
\hline & \multicolumn{10}{c}{ Xorg } \\
\hline 0.01 & 0.035 & 0.075 & 0.121 & 0.117 & 0.243 & 0.035 & 0.075 & 0.121 & 0.117 & 0.243 \\
0.02 & 2.5035 & 4.4695 & 6.6966 & 8.2255 & 9.7695 & -0.8102 & -1.4964 & -2.1054 & -2.9105 & -3.3467 \\
0.03 & 2.0921 & 3.4918 & 5.0941 & 6.1076 & 7.8581 & -0.4100 & -1.1792 & -1.5808 & -2.3949 & -2.7874 \\
0.04 & 1.5133 & 2.4726 & 3.4826 & 4.8721 & 6.5100 & -0.1901 & -0.8122 & -1.2352 & -1.8191 & -2.0885 \\
0.05 & 0.5252 & 1.4043 & 1.6639 & 3.5932 & 5.3940 & -0.0853 & -0.5834 & -1.0454 & -1.5682 & -1.8506 \\
\hline
\end{tabular}

Sodium nitroprusside + DMSO + water

\begin{tabular}{lcccccccccc}
\hline & \multicolumn{10}{c}{ Xorg } \\
\hline 0.01 & 0.027 & 0.059 & 0.097 & 0.144 & 0.201 & 0.027 & 0.059 & 0.097 & 0.144 & 0.201 \\
0.02 & 3.1195 & 4.2045 & 5.4575 & 7.1265 & 8.2988 & -1.0192 & -1.7471 & -2.3732 & -3.4993 & -3.8276 \\
0.03 & 1.5396 & 2.7276 & 3.8456 & 4.8761 & 6.0566 & -0.4402 & -1.0689 & -1.7329 & -2.3919 & -3.0861 \\
0.04 & 1.0057 & 1.8100 & 2.5527 & 4.2513 & 4.8103 & -0.2337 & -0.6731 & -1.1157 & -1.5248 & -1.9574 \\
0.05 & 0.5917 & 1.3447 & 1.8991 & 2.4220 & 2.6882 & -0.1255 & -0.4550 & -0.7870 & -1.1165 & -1.4233 \\
\hline & 0.3122 & 0.5718 & 1.5053 & 1.8407 & 2.1369 & -0.0968 & -0.3423 & -0.5879 & -0.8513 & -1.0906 \\
\hline
\end{tabular}

Sodium nitroprusside + n-propanol + water

\begin{tabular}{lccccccccccc}
\hline & \multicolumn{10}{c}{ Xorg } \\
\hline 0.01 & 0.026 & 0.057 & 0.094 & 0.138 & 0.194 & 0.026 & 0.057 & 0.094 & 0.138 & 0.194 \\
0.02 & 5.9045 & 3.6885 & 2.5045 & 1.8966 & 1.2107 & 0.7716 & 2.3623 & 3.0625 & 3.4633 & 4.4763 \\
0.03 & 4.1491 & 2.5066 & 1.7535 & 1.4344 & 0.7427 & 0.4903 & 1.6857 & 2.2358 & 2.7212 & 3.1981 \\
0.04 & 3.1457 & 1.7097 & 1.5021 & 0.8798 & 0.3870 & 0.3933 & 1.2993 & 1.5603 & 2.0859 & 2.6904 \\
0.05 & 1.8364 & 1.0602 & 0.6956 & 0.5131 & 0.2495 & 0.3473 & 0.7949 & 1.2099 & 1.6451 & 2.0475 \\
\hline
\end{tabular}




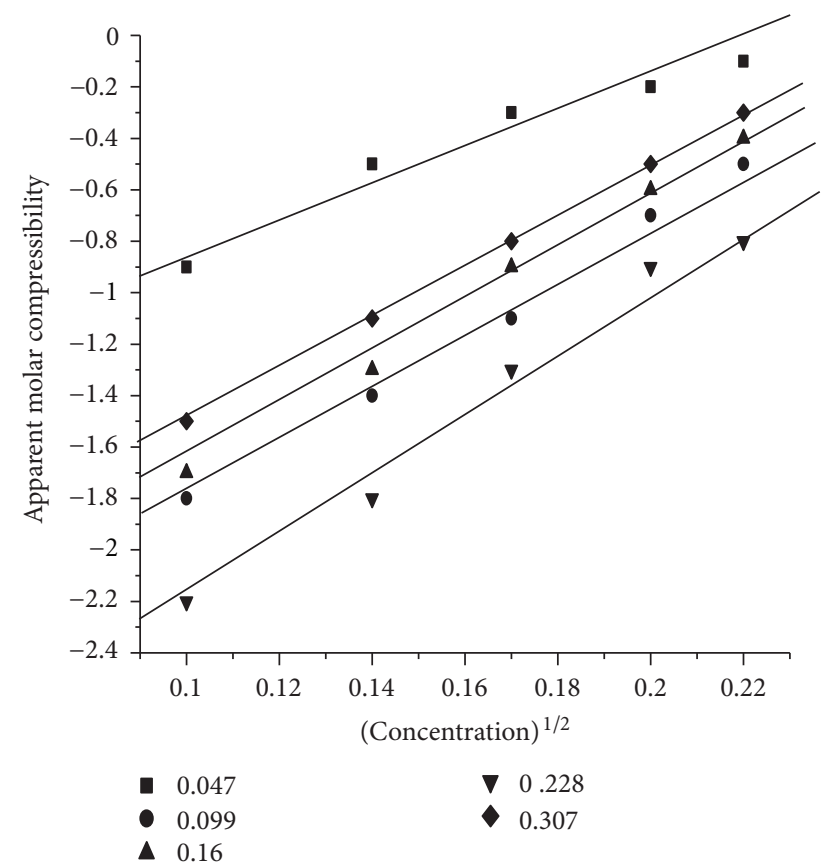

FIGURE 1: Variation of apparent molar compressibility $\left(K_{\phi}\right) C^{1 / 2}$ for sodium nitroprusside in different nitroprusside mole fraction of $\mathrm{CH}_{3} \mathrm{OH}$ in water at $308.15 \mathrm{~K} . \mathrm{K}_{\phi}^{0}$ corresponds to its value at zero concentration.

The decrease in relative association $\left(R_{A}\right)$ is due to the breaking up of the solvent molecules on adding the solute, whereas the increase as $R_{A}$ may be due to the solvation of solute [21]. In all the systems, $R_{A}$ increases with the increase in the concentration due to the increase in electrostatic attraction. From this data, it can be said that ion-ion interaction between nitroprusside ions overcomes the ionsolvent interaction.

The following observations have been made on $K_{\phi}$ and $V_{\phi}$ of sodium nitroprusside in aqueous $\mathrm{CH}_{3} \mathrm{OH}$, ethylene glycol, DMSO, and n-propanol mixtures whose values are given in Table 2.

(i) The values of $K_{\phi}$ are negative for all the systems over the entire range of concentration.

(ii) The values of $K_{\phi}$ increase linearly with increasing the ion concentration.

(iii) The values of $V_{\phi}$ are positive for $\mathrm{CH}_{3} \mathrm{OH}$ and $\mathrm{n}$ propanol whereas negative for ethylene glycol and DMSO.

Further, the negative values of $V_{\phi}$ indicated electrostatic solvation of ions [22]. In other words, the large negative value of $K_{\phi}$ may indicate the presence of packing or caging effect [23]. The decrease in the negative value indicates that with the increase in the mole fraction of aquo-organic solvents, ionsolvent interaction increases as the caging effect diminishes.

The limiting apparent molar compressibility $K_{\phi}^{0}$ provides information regarding ion-solvent interaction. The values of limiting apparent molar compressibility $K_{\phi}^{0}$ are negative in all the systems as the shown in Figures 1, 2, 3, and 4. It

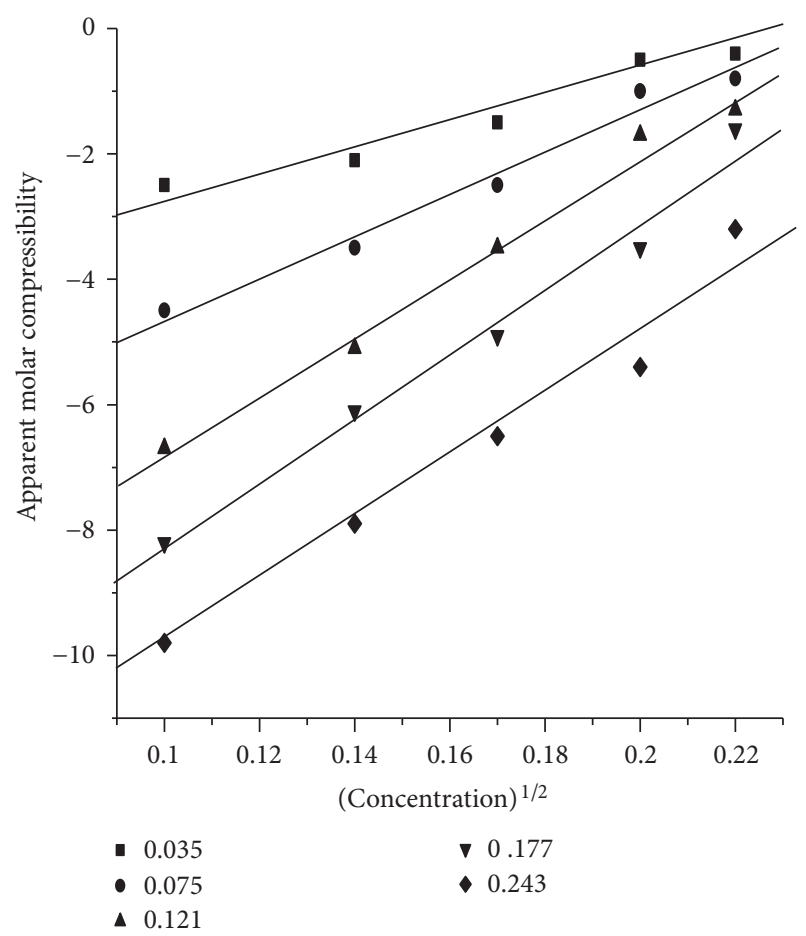

FIGURE 2: Variation of apparent molar with compressibility $\left(K_{\phi}\right)$ with $C^{1 / 2}$ for sodium in different mole fraction of ethylene glycol in water at $308.15 \mathrm{~K} . K_{\phi}^{0}$ corresponds to its value at zero concentration.

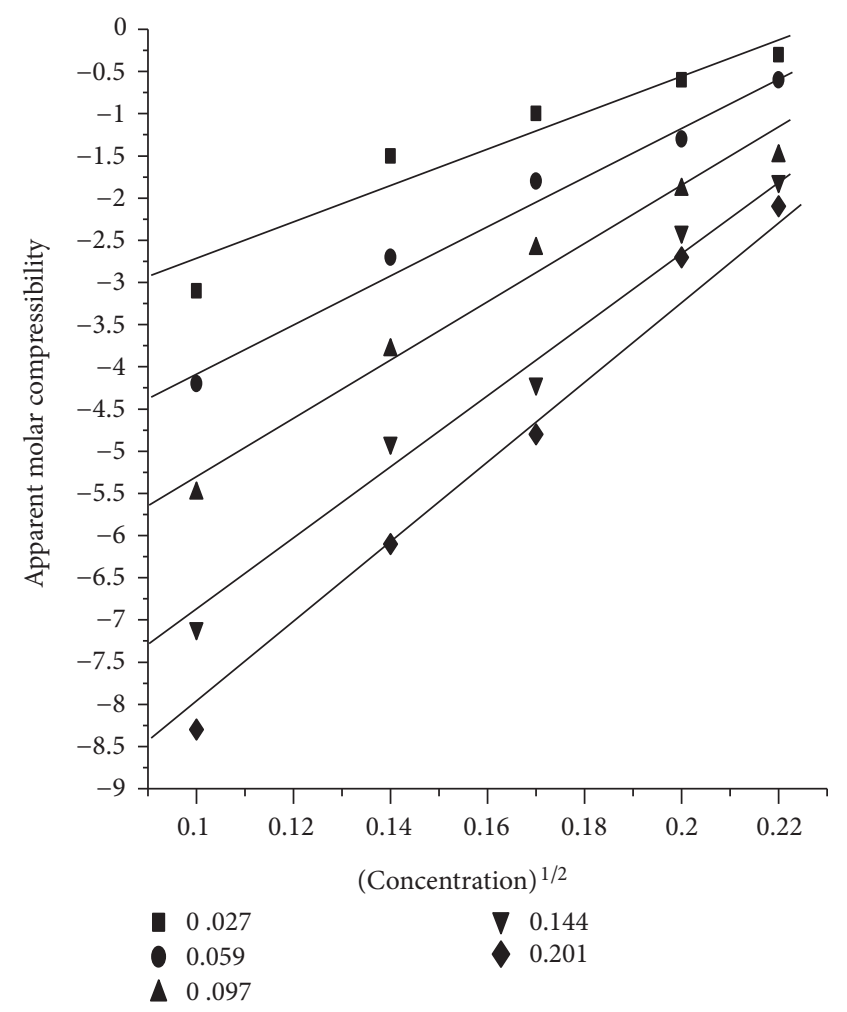

FIGURE 3: Variation of apparent molar compressibility $\left(K_{\phi}\right)$ with $C^{1 / 2}$ for sodium nitroprusside in different nitroprusside in different mole fraction of DMSO in water at $308.15 \mathrm{~K} . K_{\phi}^{0}$ corresponds to its value at zero concentration. 
TABLE 3: Results of limiting apparent molar compressibility $\left(K_{\phi}^{0}\right)$, limiting apparent molar volume $\left(V_{\phi}^{0}\right)$, and $S_{K}$ and $S_{V}$.

\begin{tabular}{|c|c|c|c|c|}
\hline Xorg & $\begin{array}{l}\text { Limiting apparent molar compressibility } \\
\qquad\left(K_{\phi}^{0}\right) \mathrm{m}^{2} \mathrm{~N}^{-1}\end{array}$ & $\begin{array}{l}\text { Limiting apparent molar volume } \\
\qquad\left(V_{\phi}^{0}\right) \mathrm{m}^{3} \mathrm{~mol}^{-1}\end{array}$ & $\begin{array}{c}S_{K} \\
\mathrm{~m}^{3} \mathrm{~mol}^{-2} \mathrm{~kg} \mathrm{~Pa}^{-1}\end{array}$ & $\begin{array}{c}S_{V} \\
\mathrm{~m}^{3} \mathrm{~kg}^{1 / 2} \mathrm{~mol}^{-3 / 2}\end{array}$ \\
\hline \multicolumn{5}{|c|}{ Sodium nitroprusside $+\mathrm{CH}_{3} \mathrm{OH}+$ water } \\
\hline 0.047 & -0.94 & 1.1 & 0.291 & -0.123 \\
\hline 0.099 & -1.86 & 1.85 & 0.383 & -0.194 \\
\hline 0.160 & -1.72 & 2.75 & 0.404 & -0.306 \\
\hline 0.228 & -2.26 & 3.5 & 0.424 & -0.384 \\
\hline 0.307 & -1.58 & 4.6 & 0.383 & -0.510 \\
\hline \multicolumn{5}{|c|}{ Sodium nitroprusside + ethylene glycol + water } \\
\hline 0.035 & -3.0 & -0.9 & 0.176 & 0.172 \\
\hline 0.075 & -4.1 & -1.57 & 0.306 & 0.268 \\
\hline 0.121 & -7.4 & -2.26 & 0.424 & 0.384 \\
\hline 0.177 & -8.8 & -2.98 & 0.466 & 0.474 \\
\hline 0.243 & -10.2 & -3.53 & 0.488 & 0.577 \\
\hline \multicolumn{5}{|c|}{ Sodium nitroprusside + DMSO + water } \\
\hline 0.027 & -2.9 & -0.95 & 0.249 & 0.176 \\
\hline 0.059 & -4.35 & -1.8 & 0.344 & 0.268 \\
\hline 0.097 & -5.56 & -2.6 & 0.404 & 0.364 \\
\hline 0.144 & -7.25 & -3.7 & 0.488 & 0.445 \\
\hline 0.201 & -8.45 & -3.85 & 0.554 & 0.532 \\
\hline \multicolumn{5}{|c|}{ Sodium nitroprusside $+\mathrm{n}$-propanol + water } \\
\hline 0.026 & -6.0 & 0.8 & 0.554 & -0.466 \\
\hline 0.057 & -3.8 & 2.3 & 0.384 & -0.404 \\
\hline 0.094 & -2.8 & 3.0 & 0.287 & -0.344 \\
\hline 0.138 & -1.9 & 3.65 & 0.213 & -0.268 \\
\hline 0.194 & -1.2 & 4.4 & 0.141 & -0.105 \\
\hline
\end{tabular}

decreases with the increase the in concentration of sodium nitroprusside solution for ethylene glycol and DMSO systems but increases in case of $n$-propanol system. The decrease is not regular in $\mathrm{CH}_{3} \mathrm{OH}$ system. It implies that a little bit ionion interaction exists in $\mathrm{CH}_{3} \mathrm{OH}$ and more ion-ion interaction in n-propanol systems. But the large negative values for ethylene glycol and DMSO systems indicate negligible ionion interaction and stronger ion-solvent interaction.

The volume behavior of solute at infinite dilution is satisfactorily represented by $V_{\phi}^{0}$ which is independent of ion-ion interaction and provides information concerning ion-solvent interaction. The values of $V_{\phi}^{0}$ are positive for $\mathrm{CH}_{3} \mathrm{OH}$ and n-propanol shown in Figures 5 and 8, whereas these are negative for ethylene glycol and DMSO (Figures 6 and 7).Thus in ethylene glycol and DMSO solutions, predominance of ion-solvent interaction is again proved by negative $K_{\phi}^{0}$ and $V_{\phi}^{0}$ values. However, in $\mathrm{CH}_{3} \mathrm{OH}$ and npropanol solutions, positive values of $V_{\phi}^{0}$ suggest that ion-ion interactions predominate.

The constant $S_{K}$ provides information regarding ion-ion interaction. The values of $S_{K}$ (Table 3) exhibit positive in all the systems. These values increase in ethylene glycol and DMSO systems, but this increase is not regular in $\mathrm{CH}_{3} \mathrm{OH}$ confirming insignificant ion-ion interaction. It decreases in n-propanol system. The value of $S_{K}$ in n-propanol system is higher at low mole fraction, indicating more ion-ion interaction. In case of ethylene glycol and DMSO systems, ion-ion interactions are stronger because of having greater value of $S_{K}$. So ion-ion interaction is favoured at high mole fraction of ethylene glycol and DMSO, but it is favoured at low mole fraction of n-propanol. The $S_{v}$ exhibits negative values in $\mathrm{CH}_{3} \mathrm{OH}$ and n-propanol systems suggesting the presence of more ion-ion interactions and less complex formation occurring in the systems. The values of $S_{v}$ which exhibit positive from Table 3 in ethylene glycol and DMSO systems reflect the existence of weak ion-ion interactions and predict the complex formation taking place in the systems.

Generally, the types of interactions occurring between sodium nitroprusside and aqueous solutions of solvents can be classified as follows:

(i) ionic-hydrophilic interactions between the ions of sodium nitroprusside and polar group of solvents,

(ii) hydrophilic-hydrophobic interactions between the ions of sodium nitroprusside and the nonpolar side group of solvents.

\section{Conclusion}

In summary, volume and compressibility data have been determined for sodium nitroprusside in aqueous $\mathrm{CH}_{3} \mathrm{OH}$, 


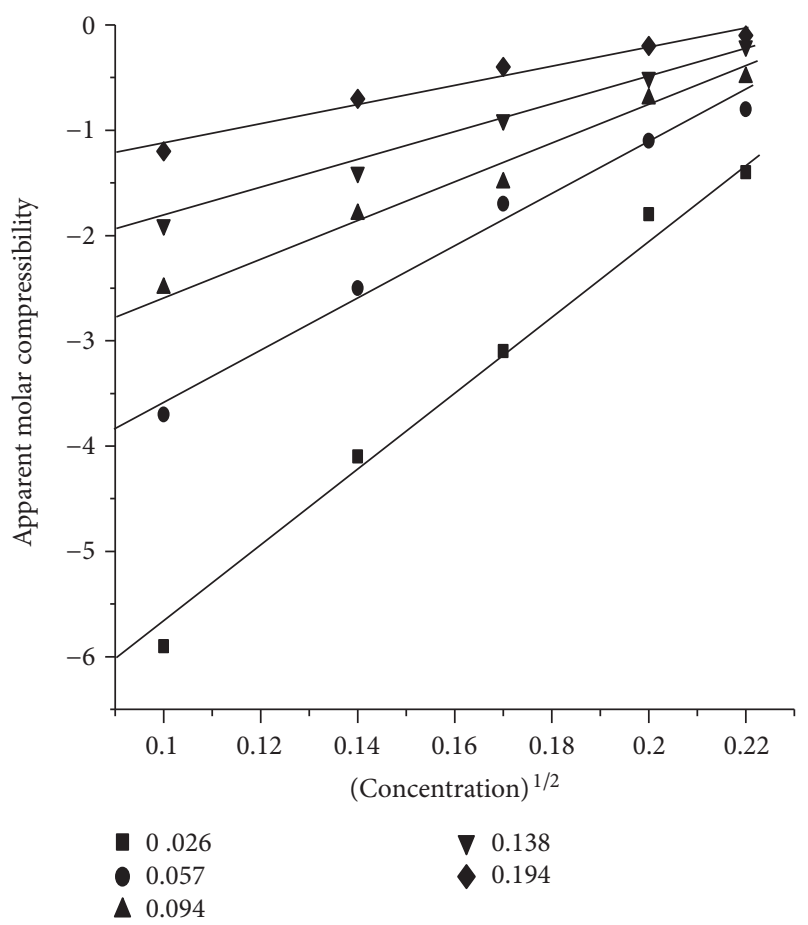

FIGURE 4: Variation of apparent molar compressibility $\left(K_{\phi}\right)$ with $C^{1 / 2}$ for sodium nitroprusside in different mole fraction of $n$ propanol in water at $308.15 \mathrm{~K} . K_{\phi}^{0}$ corresponds to its value at zero concentration.

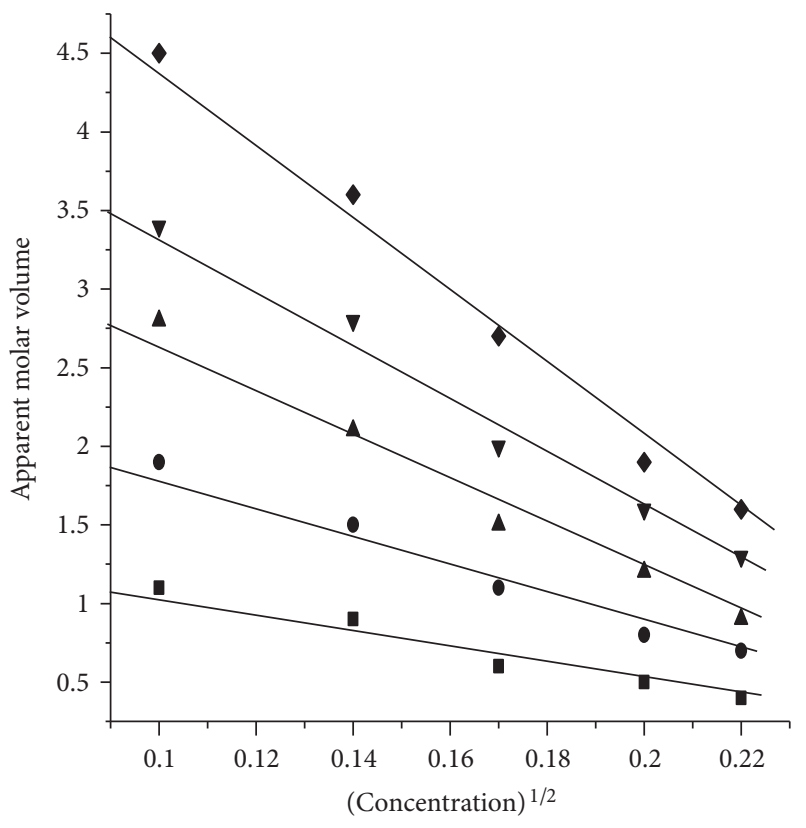

- 0.047

$\boldsymbol{\nabla} 0.228$

0.099

0.307

0.16

FIGURE 5: Variation of apparent molar volume $\left(V_{\phi}\right)$ with $C^{1 / 2}$ for sodium nitroprusside in different mole fraction of $\mathrm{CH}_{3} \mathrm{OH}$ in water at $308.15 \mathrm{~K}$. $V_{\phi}^{0}$ corresponds to its value at zero concentration.

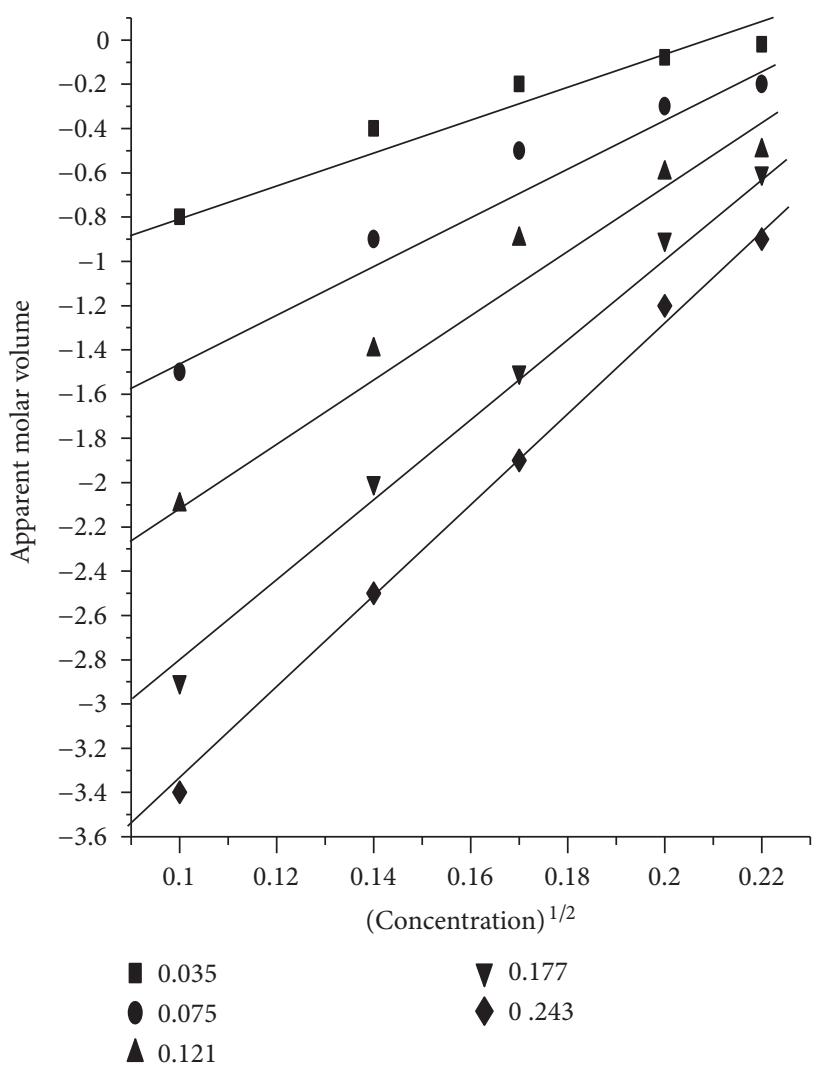

FIGURE 6: Variation of apparent molar volume $\left(V_{\phi}\right)$ with $C^{1 / 2}$ for sodium nitroprusside in different mole fraction of ethylene glycol in water at $308.15 \mathrm{~K} . V_{\phi}^{0}$ corresponds to its value at zero concentration.

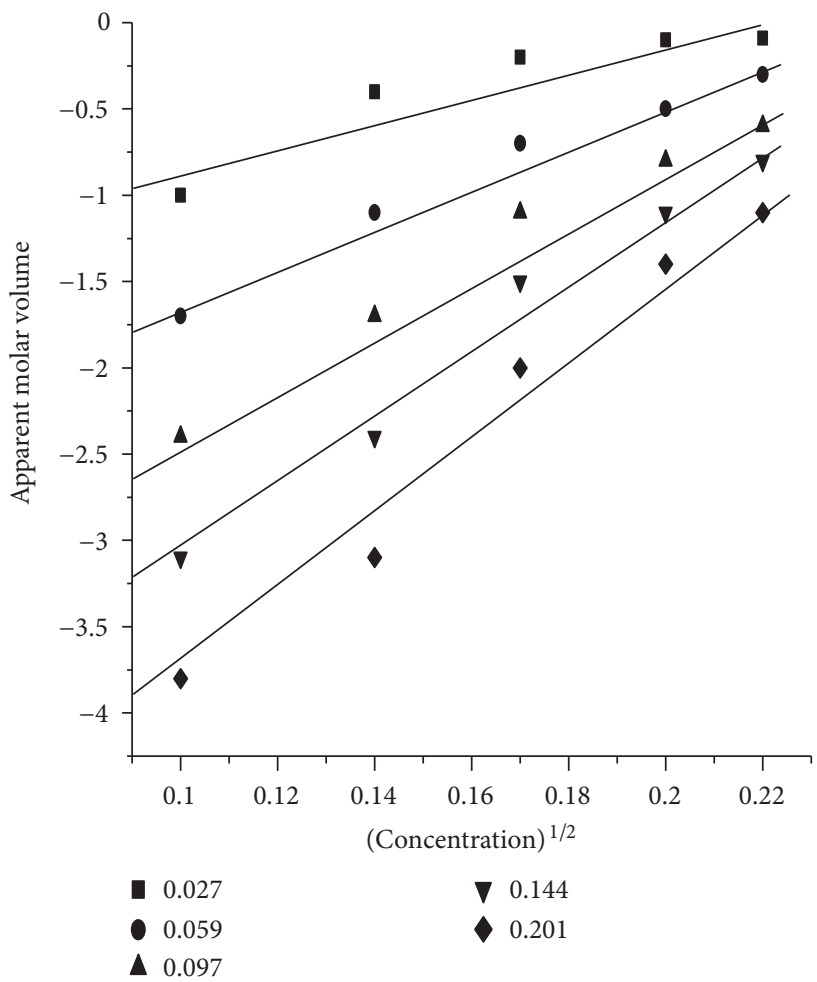

FIGURE 7: Variation of apparent molar volume $\left(V_{\phi}\right)$ with $C^{1 / 2}$ for sodium nitroprusside in different mole fraction of DMSO in water at $308.15 \mathrm{~K} . V_{\phi}^{0}$ corresponds to its value at zero concentration. 


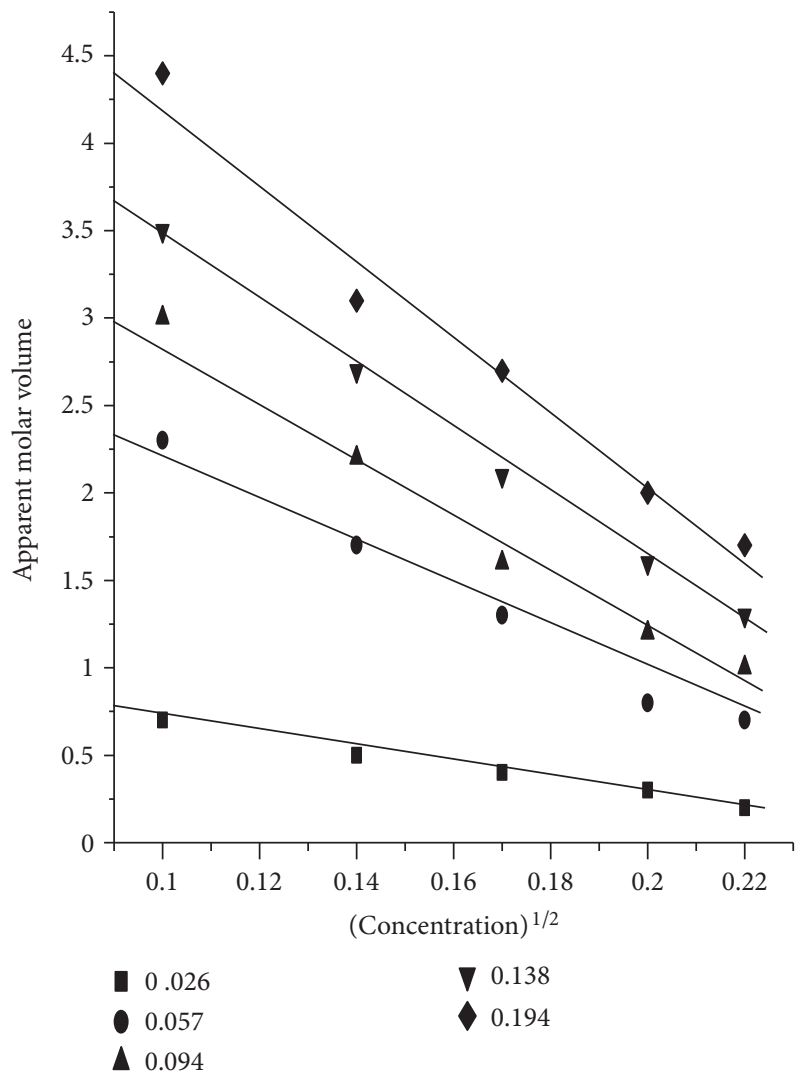

FIGURE 8: Variation of apparent molar volume $\left(V_{\phi}\right)$ with $C^{1 / 2}$ for sodium nitroprusside in different mole fraction of $\mathrm{n}$-propanol in water at $308.15 \mathrm{~K} . V_{\phi}^{0}$ corresponds to its value at zero concentration.

ethylene glycol, DMSO, and n-propanol at $308.15 \mathrm{~K}$, and the results have been used to study the ion-solvent interactions present in the mixtures. From the magnitude of $K_{\phi}^{0} \cdot V_{\phi}^{0}$, $S_{K}$ and $S_{V}$, it can be concluded that a larger ion-solvent interaction will exist in ethylene glycol and DMSO solvents than other solvents. Thus, sodium nitroprusside is an effective structure maker in aqueous ethylene glycol and DMSO solutions over other two systems.

\section{Acknowledgment}

The authors are thankful to PG Department of Chemistry, Ravenshaw University for providing facilities to carry out this work.

\section{References}

[1] S. Balija and S. Oza, "Ultrasonic studies of some derivatives of sulphonamide in dimethylformamide," Fluid Phase Equilibria, vol. 200, pp. 11-18, 2002.

[2] M. K. Rawat and Sangeeta, "Ultrasonic study of molecular interactions and compressibility behaviour of strontium soaps in chloroform-propylene glycol mixture," Indian Journal of Pure and Applied Physics, vol. 46, no. 3, pp. 187-192, 2008.

[3] A. Ali and A. K. Nain, "Ultrasonic study of molecular interactions in N,N-dimethylacetamide + ethanol binary mixtures at various temperatures," Acoustics Letters, vol. 19, pp. 181-187, 1996.

[4] H. Ogawa and S. Murakami, "Excess volumes, isentropic compressions, and isobaric heat capacities for methanol mixed with other alkanols at $25^{\circ}$ C," Journal of Solution Chemistry, vol. 16, no. 4, pp. 315-326, 1987.

[5] M. Das, S. Das, and A. K. Pattanaik, "Ultrasonic studies of cis and trans $\mathrm{K}\left[\mathrm{Cr}(\mathrm{OX})_{2}\left(\mathrm{H}_{2} \mathrm{O}\right)_{2}\right] 3 \mathrm{H}_{2} \mathrm{O}$ in ethylene glycol $+\mathrm{H}_{2} \mathrm{O}$, $\mathrm{CH}_{3} \mathrm{OH}+\mathrm{H}_{2} \mathrm{O}$ and 2-propanol $+\mathrm{H}_{2} \mathrm{O}$ at $298.15 \mathrm{~K}$," International Journal of Teaching and Research in Chemistry, vol. 18, pp. 31-41, 2011.

[6] A. P. Mishra and D. K. Mishra, "Study of Intermolecular Interaction of some synthesized $3 \mathrm{~d}$ metal complexes in solution, by ultrasonic velocity measurement," Journal of Chemical and Pharmaceutical Research, vol. 3, no. 3, pp. 489-48, 2011.

[7] W. J. Crooks and J. D. Christian, "Densities and partial molal volumes of sodium tetrafluoroborate aqueous solutions at $20^{\circ}$ C, Indian Journal of Chemistry, vol. 43, pp. 1872-1875, 2004.

[8] E. G. Panenda, P. Guardado, and A. Maestre, "Limiting partial molar volumes of electrolytes in 2-methyl-2-butanol + water mixtures at 298.15 K," Journal of Solution Chemistry, vol. 33, no. 10, pp. 1277-1293, 2004.

[9] C. W. Zhao, P. S. Ma, and J. D. Li, "Partial molar volumes and viscosity $B$-coefficients of arginine in aqueous glucose, sucrose and l-ascorbic acid solutions at $T=298.15 \mathrm{~K}$," The Journal of Chemical Thermodynamics, vol. 37, no. 1, pp. 37-42, 2005.

[10] M. L. Parmer and R. C. Thakur, "Effect of temperature on the viscosities of some divalent transition metal sulphates and magnesium sulphate in water and water+ethylene glycol mixtures," Indian Journal of Chemistry, vol. 45, pp. 1631-1637, 2006.

[11] P. S. Nikam, R. P. Shewale, A. B. Sawani, and M. Hassan, "Limiting ionic partial molar volumes and viscosities of $\mathrm{Cs}^{+}$, $\mathrm{Na}^{+},\left(\mathrm{C}_{4} \mathrm{H}_{9}\right)_{4} \mathrm{~N}^{+}, \mathrm{Cl}^{-}, \mathrm{Br}^{-}, \mathrm{I}^{-}$, and $\mathrm{BPh}_{4}{ }^{-}$in Aqueous Acetone at 308.15 K," Journal of Chemical \& Engineering Data, vol. 50, no. 2, pp. 487-491, 2005.

[12] B. J. Cox and W. E. Waghorne, "Thermodynamics of ion-solvent interactions," Chemical Society Reviews, vol. 9, pp. 381-411, 1980

[13] Y. K. Lau, P. P. S. Saluja, and P. Kebarle, "The proton in dimethyl sulfoxide and acetone. Results from gas-phase ion equilibriums involving (Me2SO)nH+ and (Me2CO)nH+," Journal of the American Chemical Society, vol. 102, no. 25, pp. 7429-7433, 1980.

[14] C. L. O’Neala, D. J. Croucha, and A. A. Fathab, "Validation of twelve chemical spot tests for the detection of drugs of abuse," Forensic Sciece International, vol. 109, no. 3, pp. 189-201, 2000.

[15] R. Rucki, "Sodium nitroprusside," in Analytical Profiles of Drug Substances, K. Florey, Ed., vol. 6, p. 487, Academic Press, New York, NY, USA, 1977.

[16] M. L. Parmer, R. K. Awasthi, and M. K. Guleria, "A study of partial molar volumes of citric acid and tartaric acid in water and binary aqueous mixtures of ethanol at various temperatures," Journal of Chemical Sciences, vol. 116, no. 1, pp. 33-38, 2004.

[17] D. D. Perrin and W. L. F. Armarego, Purification of Laboratory Chemical, Butterworth-Heinemann, 4th edition, 2000.

[18] D. Ragouramane and A. Srinivasa Rao, "Ultrasonic studies on the influence of some amino acids on molecular interactions in aqueous solutions of ethanol," Indian Journal of Chemistry, vol. 37, no. 7, pp. 659-662, 1998. 
[19] R. J. Fort and W. R. Moore, "Adiabatic compressibilities of binary liquid mixtures," Transactions of the Faraday Society, vol. 61, pp. 2102-2111, 1965.

[20] J. D. Pandey, A. Sukla, R. D. Rai, and K. J. Mishra, "Ultrasonic, volumetric, and viscometric studies of tetracycline and its allied compound," Journal of Chemical \& Engineering Data, vol. 34, no. 1, pp. 29-31, 1989.

[21] P. S. Nikam, N. Nikam, M. Hassam, and B. S. Suryawanshi, "Acoustical properties of monochloroacetic acid-acetone-water system at different temperatures," Asian Journal of Chemistry, vol. 6, no. 2, pp. 237-245, 1994.

[22] A. Dhanalakshmi and E. Vasantharani, "Analysis of apparent molal volume and apparent molal compressibility of quarternary ammonium salt in non-aqueous medium," Journal of Pure and Applied Ultrasonics, vol. 21, pp. 79-82, 1999.

[23] A. B. Wood, A Text Book of Sound, G.Bell, London, UK, 3rd edition, 1960. 

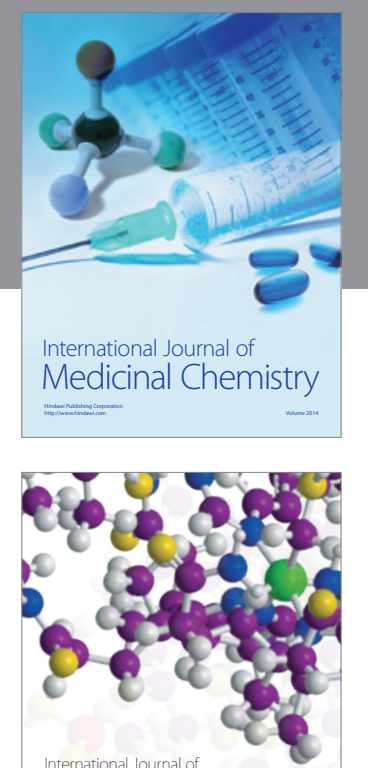

\section{Carbohydrate} Chemistry

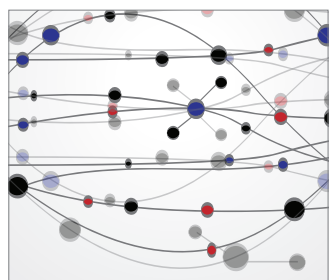

The Scientific World Journal
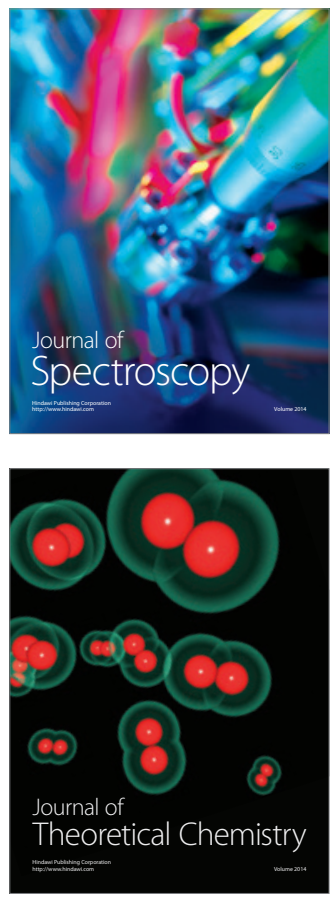
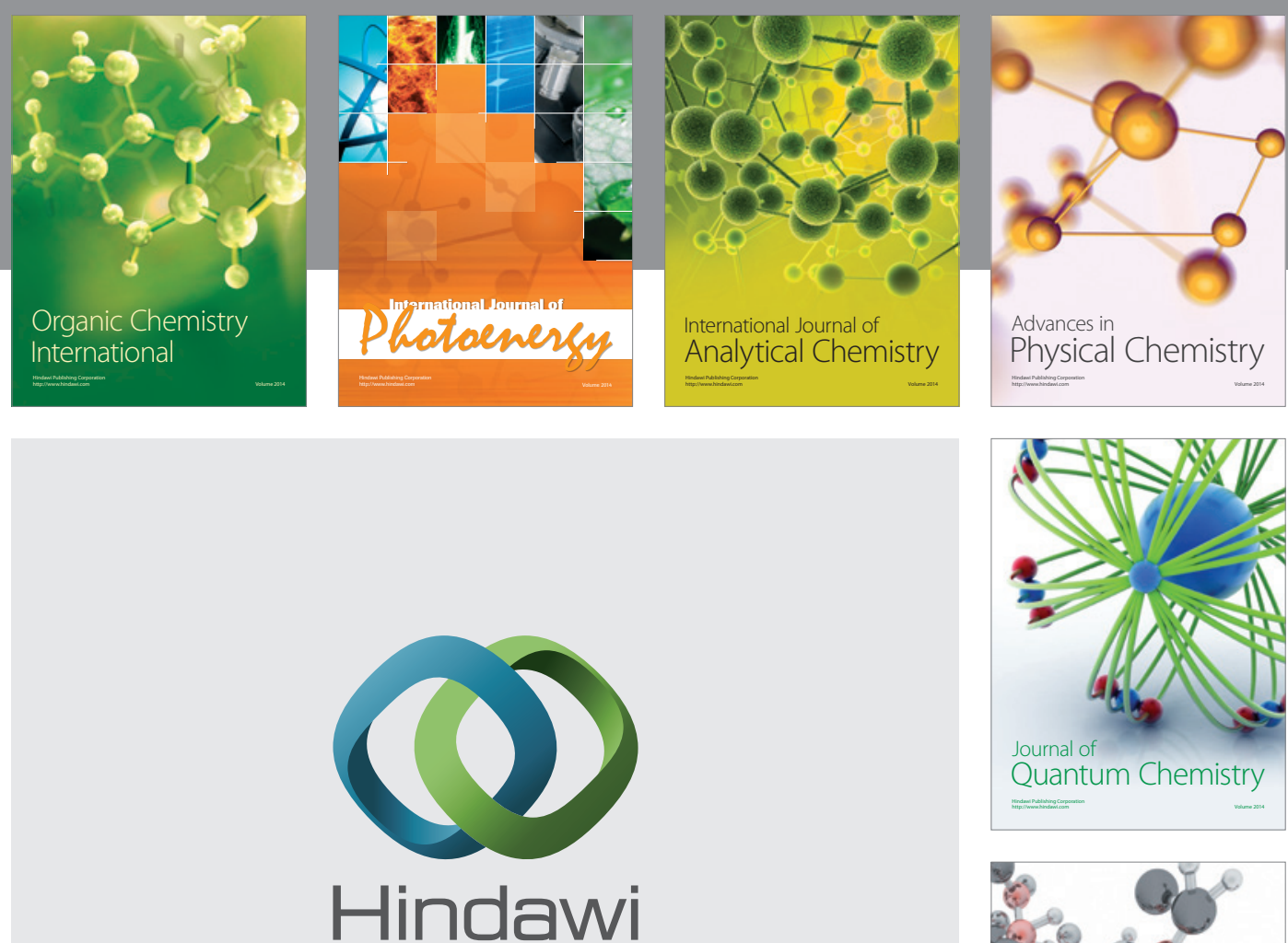

Submit your manuscripts at

http://www.hindawi.com

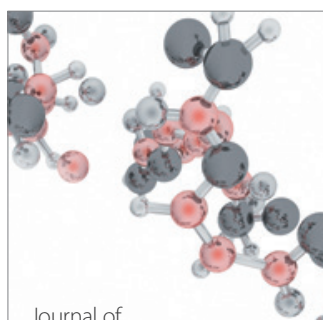

Analytical Methods

in Chemistry

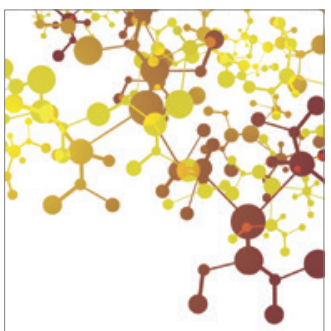

Journal of

Applied Chemistry

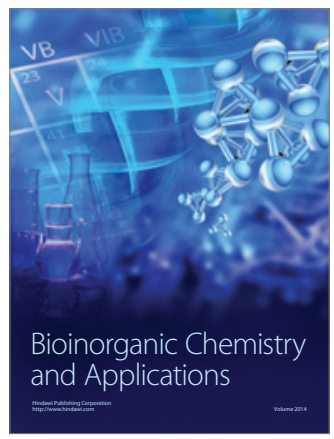

Inorganic Chemistry
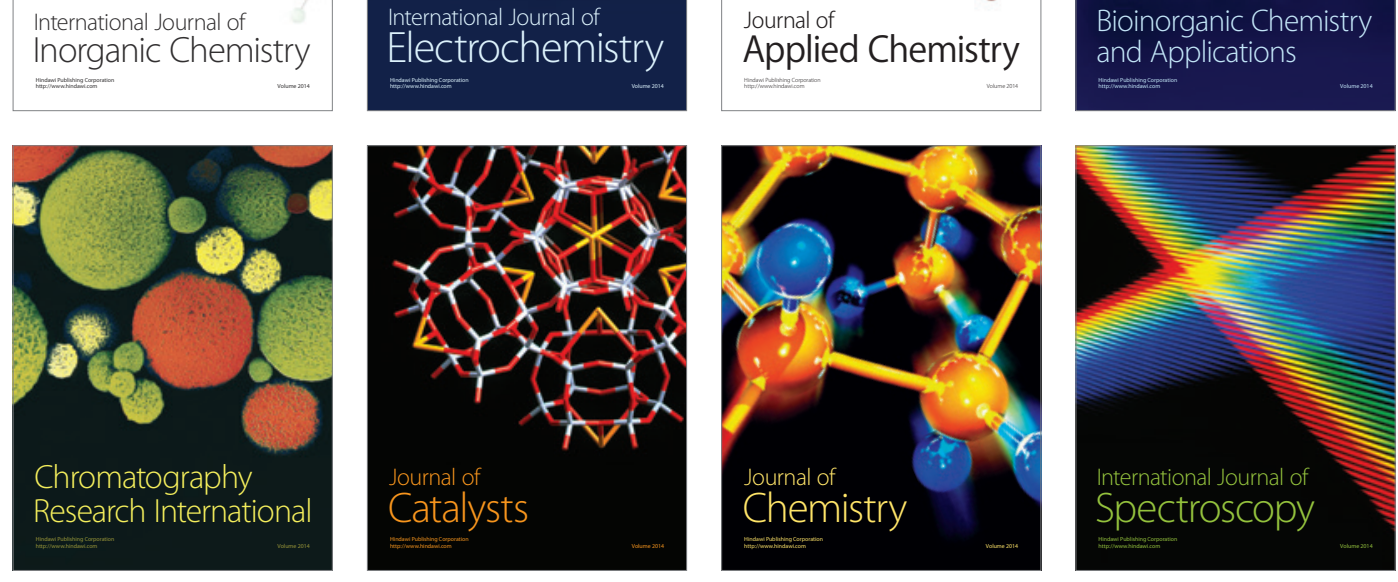